\title{
Urabstimmung FMH Services
}

Für die Verwaltung des FMH Services: Dr. med. J. de Haller, Präsident; Dr. med. M. Giger, Vizepräsident

\section{Einleitung}

Wie auch im vergangenen Jahr hat die Verwaltung des FMH Services beschlossen, die statutarischen Geschäfte (Abnahme des Jahresberichtes, Genehmigung der Rechnung 2004 sowie Entlastung der Verwaltung) einer Urabstimmung zu unterziehen. Die notwendigen Unterlagen sind in dieser Nummer der Schweizerischen Ärztezeitung publiziert. Die genauen Modalitäten sind nachfolgend beschrieben.

\section{Wer ist stimmberechtigt?}

Stimmberechtigt sind alle FMH-Mitglieder, welche vor dem 31. Dezember 2003 in die FMH als ordentliches oder ausserordentliches Mitglied aufgenommen worden sind. Weiter alle FMHMitglieder, welche sich beim Eintritt in die FMH auch zum Beitritt in die Genossenschaft FMH Services entschlossen haben.

\section{Wie wird abgestimmt?}

Alle Unterlagen für die Urabstimmung sind nachfolgend publiziert. Alle Anträge sind auf einem separaten Stimmzettel zusammengefasst. Der ausgefüllte Stimmzettel wird im Stimmcouvert in das beiliegende Rückantwortcouvert gesteckt und an die bezeichnete Treuhandstelle geschickt. Um gültig zu stimmen, ist es wichtig, das Rückantwortcouvert mit Praxisstempel und persönlicher Unterschrift zu versehen. Nur so kann gewährleistet werden, dass Genossenschaftsmitglieder ihre Stimme abgeben.

\section{Welche Frist ist zu beachten?}

Die Stimmzettel müssen bis spätestens 30. August 2005 bei der Treuhandstelle eingetroffen sein.

\section{Wer erteilt Auskünfte?}

Für Fragen steht Ihnen das Sekretariat der FMH Services, Simone Köpfli, unter der Telefonnummer 0419250077 gerne zu Ihrer Verfügung. 


\title{
Votation par correspondance FMH Services
}

\author{
Pour l'administration de FMH Services: Dr J. de Haller, Président; Dr M. Giger, Vice-président
}

\section{Introduction}

A l'instar de l'année précédente, l'administration de FMH Services a décidé de soumettre les affaires statutaires (approbation du rapport annuel et des comptes 2004, ainsi que l'attribution d'une décharge à l'administration) à une votation générale. Les documents nécessaires sont publiés dans le présent numéro du Bulletin des médecins suisses. Les modalités de détail figurent ci-après.

\section{Qui a le droit de vote?}

Tous les membres FMH ont le droit de vote qui ont été admis comme membres ordinaires ou extraordinaires avant le 31 décembre 2003, ainsi que tous les membres FMH qui lors de leur entrée à la FMH ont souhaité d'acquérir aussi la qualité d'associé de la société FMH Services.

\section{Comment la votation se déroule-t-elle?}

Tous les documents nécessaires pour la votation sont publiés ci-dessous. Un bulletin de votation contient l'ensemble des propositions. Le bulletin complété sera mis dans l'enveloppe de votation et celle-ci dans l'enveloppe réponse ci-jointe qui sera ensuite envoyée à l'agence fiduciaire indiquée. Pour que le vote soit valable l'enveloppe réponse doit être pourvue de cachet officiel et de signature personnelle. Ceci pour assurer que seuls les associés ne participent à la votation.

\section{Quels sont les délais?}

Les bulletins de votation doivent arriver à l'agence fiduciaire le 30 août 2005 au plus tard.

\section{Qui peut donner des renseignements?}

Le secrétariat de FMH Services, Simone Köpfli, sera heureux de répondre à vos questions. Son numéro de téléphone est 0419250077. 